\title{
Rezension „Smart Cities: Big Data, Civic Hackers, and the Quest for a New Utopia“"
}

\author{
Edy Portmann
}

Eingegangen: 11. Mai 2015 / Angenommen: 12. Mai 2015 / Online publiziert: 27. Mai 2015

(C) Springer Fachmedien Wiesbaden 2015

Townsend, Anthony M.: Smart Cities: Big Data, Civic Hackers, and the Quest for a New Utopia, ISBN 978-0-393-08287-6, W.W. Norton \& Co, New York 2013, 384 S., $€ 29.22$

Dieses leichtverständliche, englischsprachige Werk beleuchtet Herausforderungen, mit welchen (Groß-)Städte heutzutage kämpfen. Einige dieser Herausforderungen unterscheiden sich dabei nicht wesentlich von denen mit welchen bereits frühere Generationen zu kämpfen hatten: Beispiele sind Transport- und Verkehrswesen, Versorgung mit (Trink-)Wasser, Nahrung und Energie. Der Autor deckt in diesem Buch nun aber auch auf, wie diese Herausforderungen mit heutiger Technologie adressiert werden können - und sollten. Obwohl Technologie das Potential zur Lösung einer Reihe urbaner Herausforderungen bietet, nutzt er jedoch auch die Gelegenheit, auf mögliche Stolpersteine hinzuweisen. Dazu beleuchtet er die Irrwege der früheren Generationen genauer. Diese sollen als abschreckende Beispiele für die Entwicklung künftiger Städte dienen.

Obwohl mir viele vom Autor adressierte Trends bekannt sind, bin ich von der Tiefe seiner Recherchen beeindruckt. Townsend - Direktor des NYU Centers für Transportwesen und Berater für Industrie und Regierung - präsentiert weder einen banalisierenden Geschichtsabriss noch eine Verherrlichung der Zukunft. Vielmehr äußert er in den zehn Kapiteln eine differenzierte Anschauung zum Einfluss, welche Technologie bereits heute auf unser Leben hat und welche sie in Zukunft wohl noch stärker haben wird. Geschickt kombiniert er dabei eigene Erfahrung mit Interviews und Forschungsergebnissen, um ein möglichst holistisches Bild über heutige (Big)

\footnotetext{
E. Portmann $(\bowtie)$

Institut für Wirtschaftsinformatik (IWI), Universität Bern,

Engehaldenstrasse 8, CH-3012 Bern, Schweiz

E-Mail: edy.portmann@iwi.unibe.ch
} 
Data Sammlungen (Kap. 1 und 2), sowie der Einbindung der Öffentlichkeit bei deren Nutzung (z. B. für die Verbesserung von Dienstleistungen) zu zeichnen (Kap. 3 bis $10)$.

In Kap. 2 dient Townswend die dem Ubiquitous Computing entliehene Vorstellung der „Stadt als Computer“ als Grundlage für die Realisierung einer Smart City. Das kommende Jahrhundert (der Urbanisierung) sieht er dabei als letzte Chance um vom durch die industrielle Revolution ausgelösten Überfluss zu profitieren und gleichzeitig die Zerstörung unseres Planeten abzuwenden (Kap. 3). In den Kapiteln 4 und 5 erläutert Townsend anschließend zwei Wege zur Entwicklung Smarter Cities: Einerseits der Einsatz eines proprietären Betriebssystems (Top-Down), andererseits die Verwendung des offenen und verteilten Webs (Bottom-Up). Der Unterschied zwischen den zwei Wegen bildet denn auch der rote Faden durch den Rest des Buches.

Nach Kapiteln zu Datenschutz/Überwachung sowie Vergänglichkeit von Technologien, präsentieren Townsend in Kap. $6 \mathrm{ff}$ auch eine Reihe von Prinzipien, die sich mehrheitlich an urbanen Hacker (wie z. B. Anwälte oder Bürgerrechtler) richten, jedoch auch von den größeren Technologiefirmen verkraftet werden können. Unter Zuhilfenahme einer Reihe leicht fassbarer Beispiele zeigt er auf, dass bei der Entwicklung von Smart Cities sowohl inter-/transdisziplinäre Zusammenarbeit nötig wird und dabei Top-Down mit Bottom-Up-Ansätze vereinigt werden müssen (Kap. 9 und 10).

Da sich die von Townsend aufgezeigten Trends in Zukunft bestimmt noch intensivieren und, ganz im Sinne geistiger Munition für anstehende „Smart City Diskussionen“, sei dieses Buch allen Lesern ans Herz gelegt, mindestens aber all jenen empfohlen, welche sich für Technologie, für Gesellschaftspolitik oder beides interessieren. Sollte dem eiligen Leser jedoch die Zeit für die Lektüre des gesamten Werkes fehlen, empfehle ich mindestens das letzte Kapitel als Pflichtstoff. In den letzten Sätze bringt Townsend nämlich abschließend all diejenigen Punkte in Einklang, welchen sich heutige Städter bereits stellen: Mehr Rad fahren, städtische Landwirtschaft und Lebensmittelproduktion, Do-It-Yourself, Dinge reparieren usw. 